\title{
Breakdown of Corner States and Carrier Localization by Monolayer Fluctuations in Radial Nanowire Quantum Wells
}

\author{
Maximilian M. Sonner, ${ }^{\dagger, \downarrow, \nabla}$ Anna Sitek, ${ }^{*}, \S, \|, \nabla \odot$ Lisa Janker, ${ }^{\dagger,}$ Daniel Rudolph, ${ }^{\perp, \ddagger}$ Daniel Ruhstorfer, ${ }^{\perp, \ddagger}$ \\ Markus Döblinger, ${ }^{\#,+}$ Andrei Manolescu, ${ }^{\S}$ Gerhard Abstreiter, ${ }^{\perp,+}$ Jonathan J. Finley, ${ }^{\perp, \$}$

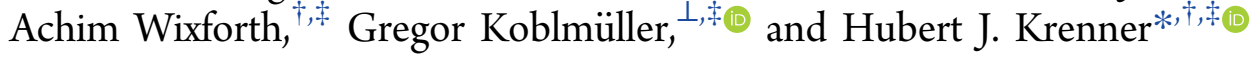 \\ ${ }^{\dagger}$ Lehrstuhl für Experimentalphysik 1, Institut für Physik and Augsburg Centre for Innovative Technologies (ACIT), Universität \\ Augsburg, Universitätsstr. 1, 86159 Augsburg, Germany \\ ${ }^{\ddagger}$ Nanosystems Initiative Munich (NIM), Schellingstr. 4, 80799 München, Germany \\ ${ }^{\S}$ School of Science and Engineering, Reykjavik University, Menntavegur 1, 101 Reykjavik, Iceland \\ "Department of Theoretical Physics, Faculty of Fundamental Problems of Technology, Wroclaw University of Science and \\ Technology, Wybrzeże Wyspiańskiego 27, 50-370 Wroclaw, Poland \\ ${ }^{\perp}$ Walter Schottky Institut and Physik Department, Technische Universität München, Am Coulombwall 4, 85748 Garching, Germany \\ \#Department of Chemistry, Ludwig-Maximilians-Universität München, Butenandtstr. 5-13(E), 81377 München, Germany
}

ABSTRACT: We report a comprehensive study of the impact of the structural properties in radial $\mathrm{GaAs}-\mathrm{Al}_{0.3} \mathrm{Ga}_{0.7}$ As nanowire-quantum well heterostructures on the optical recombination dynamics and electrical transport properties, emphasizing particularly the role of the commonly observed variations of the quantum well thickness at different facets. Typical thickness fluctuations of the radial quantum well observed by transmission electron microscopy lead to pronounced localization. Our optical data exhibit clear spectral shifts and a multipeak structure of the emission for such asymmetric ring structures resulting from spatially separated, yet interconnected quantum well systems. Charge carrier dynamics induced by a surface acoustic wave are resolved and prove efficient carrier exchange on native, subnanosecond time scales within the heterostructure. Experimental findings are corroborated by theoretical modeling, which unambiguously show that electrons and holes localize on facets where the quantum well is the thickest and that even minute deviations of the perfect hexagonal shape strongly perturb the commonly assumed 6-fold symmetric ground state.

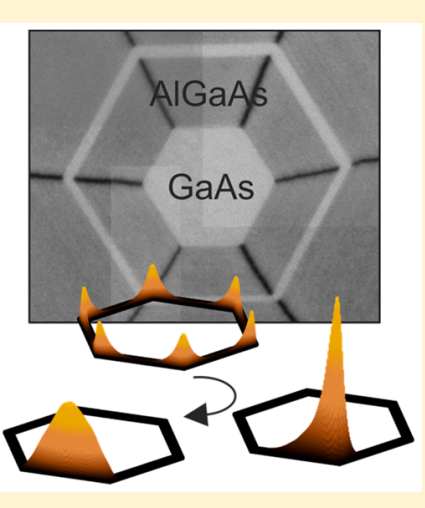

KEYWORDS: nanowires, heterostructures, fluctuations, transmission electron microscopy, surface acoustic waves,

optical recombination dynamics, electrical transport

$\mathrm{O}$ ver the past years, the paradigm of bandstructure engineering ${ }^{1}$ and the exploitation of quantum-confined systems have been transferred from planar semiconductors onto the one-dimensional platform of nanowires (NWs) $)^{2,3}$ providing building blocks for future high-performance nanoelectronic and nanophotonic devices. ${ }^{4-8}$ After first hallmark experiments, ${ }^{2,3}$ the realization of fully fledged quantum confined heterostructures such as two-dimensional quantum wells (QWs), one-dimensional quantum wires, and zerodimensional quantum dots (QDs) within the NW geometry is continuously growing and, yet, much more demanding than in conventional planar epitaxy. For example, radial coremultishell heterostructures play an important role since they allow to elegantly wrap complex QW-based and multidimensional quantum heterostructures around the typical bulk-like (3D) core of the host NW. ${ }^{9-14}$ Such advanced NW coremultishell heterostructures have already proved to be quite useful for high-performance nanowire lasers ${ }^{12,14,15}$ and lightemitting diodes, ${ }^{4} \mathrm{NW}$-based photodetectors, ${ }^{16}$ as well as novel transistors ${ }^{5,17}$ and platforms for quantum transport inves- tigations. ${ }^{13}$ However, structural disorder occurs quite commonly in QW-based heterostructures, and the role of disorder on the electrical transport ${ }^{18}$ and optical properties ${ }^{19-21}$ has been studied in great detail for planar QW heterostructures in the past.

In NWs and NW-based heterostructures, the less favorable growth conditions on the side facets of the NW lead to pronounced structural and compositional variations within ternary compounds and at interfaces of heterostructures. $^{13,22-27}$ Radial heterostructure QWs are well-known to exhibit pronounced thickness fluctuations, ${ }^{10,28}$ which, in the limit of thin QWs (thickness $\leq 4 \mathrm{~nm}$ ), even lead to the formation of "unintentional" QDs. ${ }^{29-31}$ Deviations in QW thickness may also lead to variations in the threshold and emission wavelength of QW-based NW-lasers. ${ }^{14}$ Independent

Received: March 12, 2019

Revised: April 17, 2019

Published: April 23, 2019 
of the QW thickness, deviations from a perfect hexagonal cross-section of the NW core directly transfer to the radial shell. ${ }^{10,12,32-34}$ In addition, facet-dependent growth anisotropies lead to the emergence of nontrivial extended shapes ${ }^{28}$ or the formation of nanoscale islands. ${ }^{35}$ Despite this evidence, so far most theoretical descriptions have focused almost exclusively on a symmetric hexagonal shape of the NWs. For such a perfect geometry, the ground state exhibits also 6-fold symmetry, and the wave function is delocalized between all corners of the radial QW wrapped around the hexagonal NW. ${ }^{10,33,34,36-38}$ Thus, to date only few works exist for realistic, asymmetric QW ring structure shapes. ${ }^{39-41}$ All the aforementioned commonly observed imperfections are expected to have major impact on the optical and electrical properties of NW-based heterostructures.

In this Letter, we report the impact of commonly observed

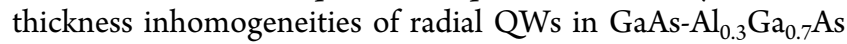
nanowire heterostructures on the optical and electronic properties. We show that typical well width fluctuations of the radial QW result in line broadening and a multipeak structure of the emission of spatially separated, yet interconnected QW systems for such tubular structures. By employing acousto-optoelectrical spectroscopy, we directly observe coupled emission dynamics of the carriers and exchange of charge carriers on subnanosecond time scales within two interconnected QW systems of the heterostructure. Moreover, theoretical calculations show that for even minute deviations of the perfect hexagonal shape of the NW-QW, electrons and holes are not confined to the corners of the radial QW but localize on the facets where the QW is the thickest. In particular, the wave function no longer distributes over the six corners of the hexagon but fully localizes on the thickest facet.

The studied core-multishell NWs were grown on a $\mathrm{SiO}_{2}$ masked $\mathrm{Si}(111)$ substrate in a solid-source molecular beam epitaxy (MBE) system via a two-step growth process. First, the GaAs core was grown in a self-catalyzed (i.e., Ga-droplet mediated) vapor-liquid-solid (VLS) growth process. ${ }^{42,43}$ Subsequently, the radial shell structure was grown in a vapor-solid growth process similar to conventional epitaxy of GaAs $/ \mathrm{Al}_{x} \mathrm{Ga}_{1-x} \mathrm{As}$ on (110) planar surfaces. ${ }^{22}$ The as-grown structure consists of a GaAs core with a diameter of $60 \mathrm{~nm}$, which is surrounded by a $30 \mathrm{~nm}$ thick $\mathrm{Al}_{x} \mathrm{Ga}_{1-x}$ As shell with a nominal Al-content of $30 \%$, followed by a $5 \mathrm{~nm}$ thick radial GaAs quantum well and a $70 \mathrm{~nm}$ thick $\mathrm{Al}_{x} \mathrm{Ga}_{1-x}$ As layer. For passivation, a GaAs capping layer of $5 \mathrm{~nm}$ has been added to prevent the oxidation of the outer $\mathrm{Al}_{x} \mathrm{Ga}_{1-x}$ As shell. The resulting heterostructure is shown schematically in Figure 1a. Furthermore, NWs were grown under conditions yielding predominantly a zinc blende (ZB) crystal structure with few twin defects and a length exceeding $10 \mu \mathrm{m}$. Further details on the growth of the heterostructure can be found elsewhere. ${ }^{22}$ Moreover, we employed spontaneous nucleation on the growth substrate without any additional prepatterning. This straightforward bottom-up approach is typically characterized by random NW nucleation and, hence, variations in the internanowire distance. In addition, such bottom-up synthesized NWs exhibit a slight tilt from the perfect vertical alignment. $^{44}$ Both effects have been found by many groups $^{11,45-47}$ and occur also for the case of our NWs, which is confirmed in the two representative scanning electron micrographs (SEM) shown in Figure 1b,c. The combination of these effects typically leads to the commonly observed characteristic inhomogeneities of the axial and radial growth (a)
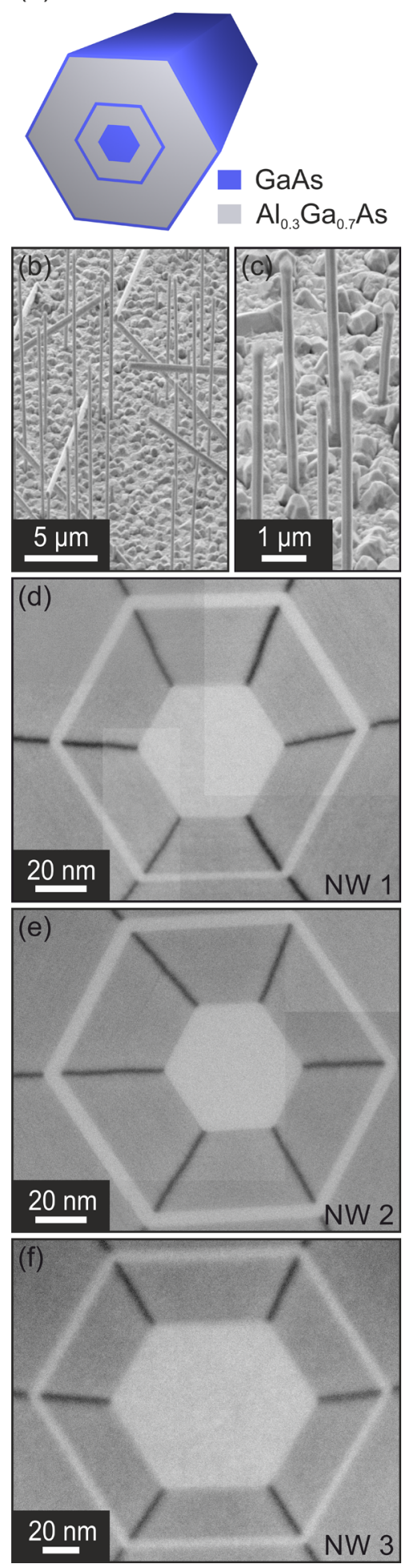

Figure 1. (a) Schematic illustration of the nanowire heterostructure consisting of a GaAs core and an $\mathrm{Al}_{0.3} \mathrm{Ga}_{0.7} \mathrm{As}$ shell with an embedded $5 \mathrm{~nm}$ thick radial GaAs quantum well $(\mathrm{QW})$. $(\mathrm{b}, \mathrm{c})$ SEM images of randomly grown GaAs- $\mathrm{Al}_{0.3} \mathrm{Ga}_{0.7}$ As core-shell NWs. (d-f) STEMHAADF cross-sectional images of the three randomly selected, representative NWs. Due to atomic number sensitive contrast of STEM-HAADF images, the GaAs QW appears brighter than the $\mathrm{Al}_{0.3} \mathrm{Ga}_{0.7} \mathrm{As}$ shell. All three NWs show clear deviations from the perfect hexagonal shape and pronounced variations of the QW thickness on different facets. 
of NWs. In particular, the radial growth of certain side facets may be partially shadowed by nearby NWs (variations in interwire distance $)^{48}$ or by the slight misorientation from exact vertical growth, ${ }^{27}$ while other facets are exposed to comparatively more growth species. This gives rise to distinct growth rate anisotropies, and hence fluctuations of the layer thicknesses on different sidewall facets, ultimately resulting in a distortion of the perfectly symmetric hexagonal cross-section of a NW. ${ }^{49-51}$ To determine the size and the microstructural properties of the asymmetric NW heterostructures we performed cross-sectional scanning transmission electron microscopy in high-angle annular dark field mode (STEMHAADF) on three randomly selected, representative NWs, labeled NW1, NW2, and NW3. The cross-sectional STEM images recorded along the [111] zone axis of three NWs are shown in Figures $1 \mathrm{~d}-\mathrm{f}$ and clearly show a deviation from a perfect hexagonal shape of the core as well as pronounced variations of the $\mathrm{QW}$ thicknesses on different facets. The obtained values are summarized in Table 1 . The mean QW

Table 1. Measured QW Thicknesses of the Asymmetric Radial QW of the Three Selected Nanowires ${ }^{a}$

$\begin{array}{ccccc} & d(\mathrm{~nm}) & \sigma(\mathrm{nm}) & d_{\max }-d(\mathrm{~nm}) & d_{\min }-d(\mathrm{~nm}) \\ \text { NW 1 } & 5.5 & 1.4 & +1.6 & -2.0 \\ \text { NW 2 } & 5.3 & 0.8 & +0.9 & -1.0 \\ \text { NW 3 } & 5.5 & 0.8 & +0.9 & -1.3\end{array}$

${ }^{a}$ Mean thickness $d$ of the radial QW is significantly larger than the nominal value of $5 \mathrm{~nm}$. The large standard deviation $\sigma$ as well as the difference between the thickest/thinnest QW facet and the mean thickness confirm pronounced inhomogeneities of the NWs' crosssection. thickness is found to be $d=5.5 \mathrm{~nm}$ or $5.3 \mathrm{~nm}$, i.e., slightly larger than the nominal value of $5 \mathrm{~nm}$. Moreover, the width of the different facets fluctuates between 3.5 and $7.1 \mathrm{~nm}$ for the NW 1. We note that for the case of all three NWs the two thickest QWs are located next to each other, whereas the thinnest QW is separated by at least one facet from the thickest one. Such inhomogeneity of the $\mathrm{QW}$ width results in large differences of the confinement energy, which has a significant impact on the emission spectrum. This detailed structural information on the obtained structures is further used to perform calculations of the electronic properties.

In the following, we investigate the optical properties of three different NWs from the same growth sample, named NW 4, NW 5, and NW 6. Figure 2a-c shows low-temperature microphotoluminescence ( $\mu$-PL) spectral maps of the NWs recorded along their axis at low optical pump power density of about $\sim 3 \frac{\mathrm{W}}{\mathrm{cm}^{2}}$. The peak centered around the energy of $1.52 \mathrm{eV}$ is observed for all three NWs and is attributed to the emission from the NW core with a predominant ZB crystal phase with only very few individual twin defects. ${ }^{52}$ Moreover, the PL of the GaAs-QW exhibits clear inhomogeneities for all three NWs. NW 4 shows a single, broad emission peak of the QW along the $\mathrm{NW}$ at an energy of about $1.58 \mathrm{eV}$. In strong contrast, the QW emission of NW 5 and NW 6 features several distinct emission bands at different energies that are spatially offset along the NW with small spatial overlapped areas. (110) planar surfaces are known to exhibit instabilities of the surface morphology resulting in the formation of islands and valleys, which reduce the optical and electrical properties. ${ }^{53}$ These well width fluctuations around and along the NWs will lead to the

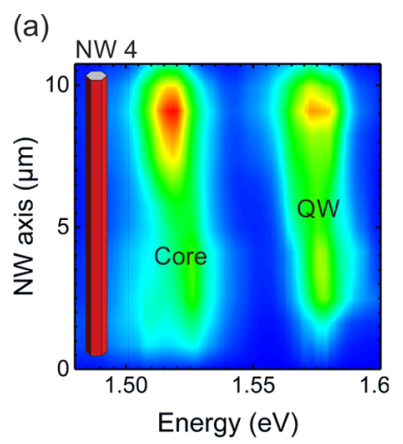

(d)
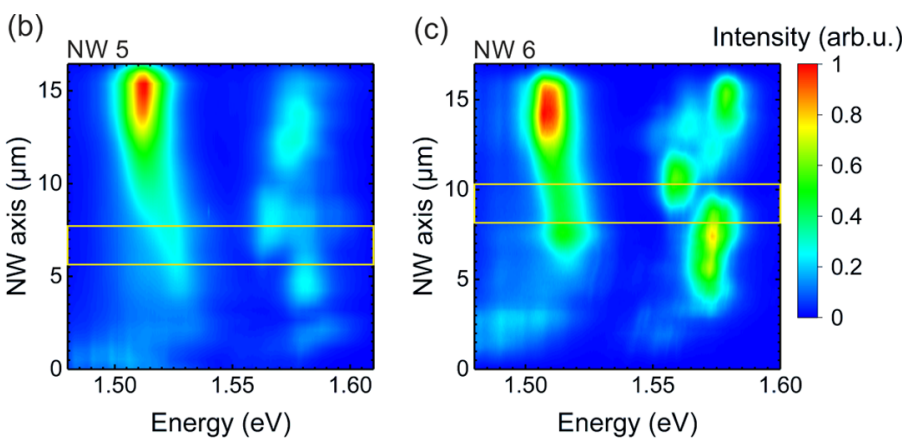

(e)
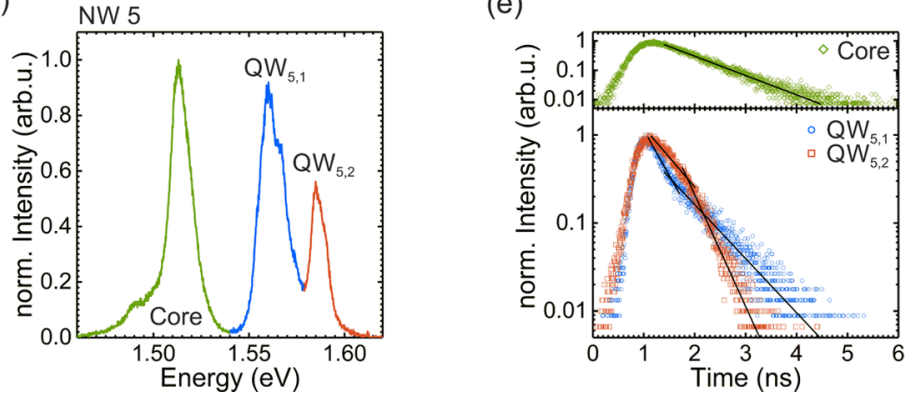

Figure 2. $(\mathrm{a}-\mathrm{c})$ PL mappings of three representative NWs. NW 4 (a) shows one broad emission energy of the QW along the NW, whereas NW 5 (b) and NW 6 (c) exhibit several different emission signals of the GaAs-QW. The regions of emission are spatially offset along the NW with a small spatial overlap area. The yellow boxes indicate the region of excitation and detection along the NW featuring the two emission centers in the signal for the measurements with SAW. (d) Time-integrated PL spectrum of NW 5 showing a double peak structure of the radial QW (attributed to the two spatially separated but interconnected QW systems with the largest thickness) and of the core. (e) Time evolution of PL intensity recorded for each peak of the PL spectrum of NW 5. The solid lines are guides to the eyes, showing an anticorrelated time evolution for both QWs suggesting a dynamic charge exchange between coupled systems. 
observed line broadening and the local emission centers. $^{19,54-56}$

Figure $2 \mathrm{~d}$ shows a selected spectrum of NW 5 at a position along the NW indicated by a yellow box in Figure $2 \mathrm{~b}$. It consists of two overlapping emission bands with energies of approximately $1.561 \mathrm{eV}$ (blue, referred as $\mathrm{QW}_{5,1}$ ) and $1.585 \mathrm{eV}$ (orange, referred as $\mathrm{QW}_{5,2}$ ) and the $\mathrm{ZB}$ core with peak centered at approximately $1.513 \mathrm{eV}$. This double peak structure of the QW originates from two spatially separated but interconnected QW systems, which exhibit the maximum thickness at their respective axial position within the radial QW structure. This we will corroborate by acoustic-optoelectrical spectroscopy and theoretical modeling. In order to probe the carrier dynamics and to identify the origin of the two emission peaks of the QW, we performed temporally resolved PL (TRPL) of the core and the radial QW, which is plotted in Figure 2e. The core exhibits the established clear monoexponential decay of an isolated system. Also, the characteristic decay time of $\sim 0.7 \mathrm{~ns}$ is well consistent with literature data for free excitons in GaAs nanowires. ${ }^{52,57,58}$ In strong contrast, we observe a clear biexponential decay for both QW emission signals. The decay time of $\mathrm{QW}_{5,1}$ increases from $\sim 0.4$ to $\sim 0.7$ $\mathrm{ns}$, whereas the decay time of $\mathrm{QW}_{5,2}$ decreases from $\sim 0.6$ to $\sim 0.3$ ns. This anticorrelated behavior, as clearly seen by the crossover of the two TRPL curves in Figure 2e, can be explained by a charge carrier transfer between two interconnected QW systems, more precisely a transfer from the higher energetic $\mathrm{QW}$ system $\mathrm{QW}_{5,2}$ toward the lower energetic $\mathrm{QW}_{5,1}$.

Next, we show that the aforementioned redistribution of carriers can be triggered and even reversed on subnanosecond time scales by the acousto-electric effect. To this end, we employ acousto-optoelectrical spectroscopy ${ }^{59,60}$ for which $\mathrm{NWs}$ were transferred from solution onto a YZ-cut $\mathrm{LiNbO}_{3}$ substrate with lithographically predefined interdigital transducer (IDTs). By applying a resonant RF signal to the IDTs, a Rayleigh-type SAW is excited with a wavelength of about $\lambda=$ $18 \mu \mathrm{m}$, which corresponds to a resonance frequency of $f_{\mathrm{SAW}}=$ $194 \mathrm{MHz}$ and acoustic period $T_{\mathrm{SAW}}=5.15 \mathrm{~ns}$. For our experiments we select NWs with their (111) growth axis being aligned parallel to the SAW's propagation direction as shown schematically in Figure 3a. When propagating on a piezoelectric substrate, the mechanical wave induces an electric field gyrating in the sagittal plane. ${ }^{24}$ For the selected power regime, the electric field dissociates photogenerated excitons and drives the dynamics of such generated electrons. In this regime, holes remain to be quasi-stationary due to their lower mobility. ${ }^{60-63}$ Further on, the SAW-driven dynamics lead to the injection and depletion of electrons to the corresponding emission region resulting in the formation and dissociation of excitons, respectively. The injection (depletion) causes an increase (decrease) of the optical signal and, correspondingly, a reduction (increase) of the total decay rate. These SAWdriven charge carrier dynamics can be resolved in a characteristic time-modulated PL transient under SAW excitation. ${ }^{60,61,64}$ In the left panels of Figure $3 b, c$, we show the time-integrated (without SAW) PL emission spectra signals of the above characterized NW 5 and NW 6. These spectra are taken at positions of two neighboring emission regions that spatially overlap along the NW (indicated in Figure $2 b, c$ ) and thus show two dominant emission peaks labeled $\mathrm{QW}_{5,1}, \mathrm{QW}_{5,2}$ and $\mathrm{QW}_{6,1} \mathrm{QW}_{6,2}$, respectively. We recorded SAW-modulated, time-resolved PL transients for each emission peak. The (a)

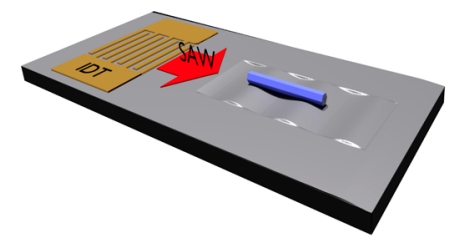

(b)

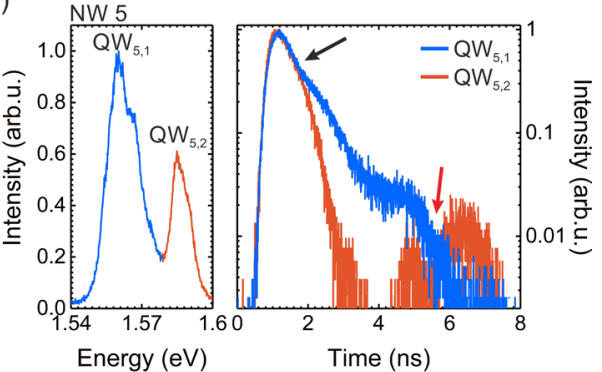

(c)

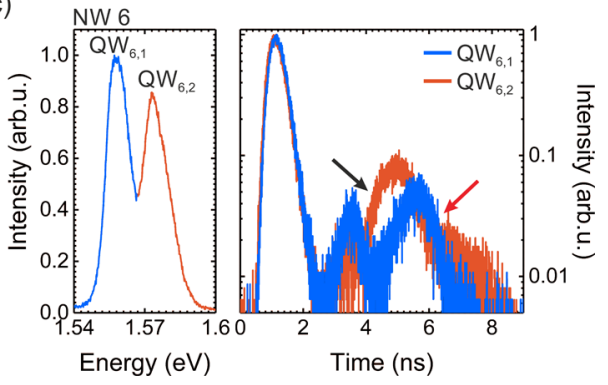

Figure 3. (a) Schematic of a typical SAW chip used for single NW acousto-optoelectrical spectroscopy. It consists of an IDT generating a SAW, which propagates toward the NW. The NW is aligned parallel to the SAW's propagation direction. The SAW creates a gyrating electric field that leads to a dissociation and acceleration of electrons and holes. $(b, c)$ Representative time-integrated and corresponding time-resolved PL emission spectra of NW 5 and NW 6 at the position with two different emission energies (blue, orange) of the two QW systems (indicated by yellow boxes in Figure $2 \mathrm{~b}, \mathrm{c}$ ). The transients exhibit an anticorrelated behavior between both QW systems (indicated by black and red arrows). This can be attributed to a dynamically triggered electron transfer between two interconnected QWs due to the gyrating electric field of the SAW, which induces an overall oscillating movement in radial direction around the hexagonal QW and in axial direction along the NW (black arrow: electron transfer from blue transient, i.e., $\mathrm{QW}_{5,1}$ or $\mathrm{QW}_{6,1}$, to orange, i.e., $\mathrm{QW}_{5,2}$ or $\mathrm{QW}_{6,2} ;$ red arrow: electron transfer from orange transient to blue).

observed transients are shown in the right panels of Figure $3 b, c$. For each NW, the two corresponding transients exhibit clear anticorrelations, which arise from SAW-induced charge carrier dynamics: at the times indicated by the black arrows, one of the two transients of each NW shows a decrease of the total decay rate of the energetically lower emission signal $\left(\mathrm{QW}_{5,1}\right.$ with respect to $\left.\mathrm{QW}_{6,1}\right)$, while simultaneously the total decay rate of the energetically higher emission feature $\left(\mathrm{QW}_{5,2}\right.$ with respect to $\left.\mathrm{QW}_{6,2}\right)$ increases. This observation points toward a simultaneous depletion of electron population of the energetically higher QW and an injection of these electrons into the energetically lower QW. Moreover, the reversed process, a transfer from energetically higher into energetically lower QW region, is observed at the times indicated by the red arrows. Thus, the observed anticorrelations can be attributed to dynamically triggered electron transfer between two interconnected QWs due to the gyrating electric field of the SAW. Most importantly, this transfer is reversible and occurs on subnanosecond time scales. 
(a)
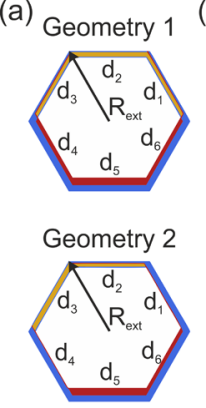

(b)
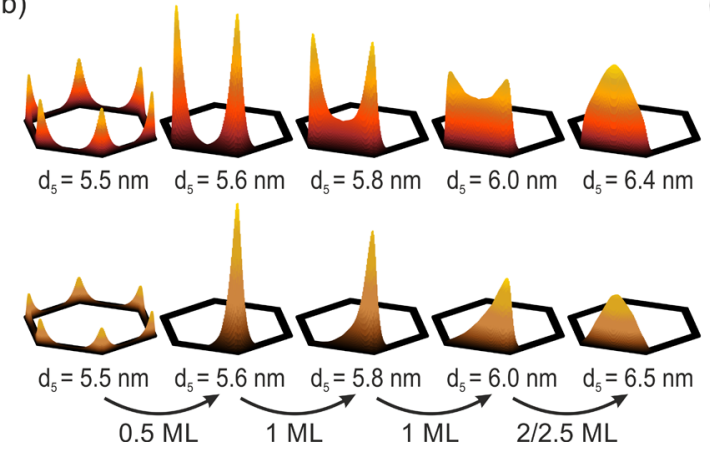

(c)

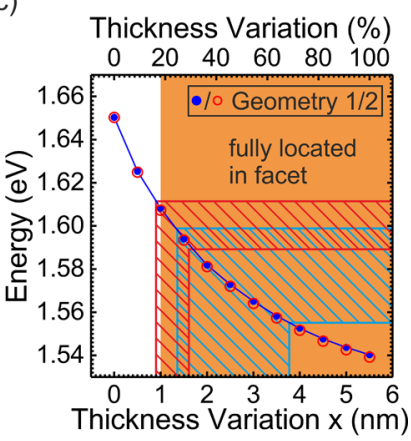

Figure 4. (a) Sample models. Geometry 1 represents the symmetric deformation of the radial QW, whereas geometry 2 describes the asymmetric case. The blue color represents the symmetric sample with $d=5.5 \mathrm{~nm}$ and $R_{\text {ext }}=80 \mathrm{~nm}$, the red color indicates the overgrowth with respect to the symmetric sample, while the yellow regions represent the parts of the sample that are subtracted from the symmetric hexagon due to the increase of the thickness variation parameter $x$. The radial QW geometries used for simulations are listed in Table 1. (b) Ground state localization for increasing the width of the thickest sidewall facet $\left(d_{5}=d+x\right)$. Increasing the thickness variation parameter $x$ leads to the breaking of the 6-fold corner states symmetry and to a transition from corner to side states. (c) Excitonic transition energies obtained with the electron-hole model (e-h model) versus QW thickness variation parameter $x$. The blue shaded box associates the experimentally obtained emission energies of the radial QW of the three NWs with the calculated side thicknesses, while the red shaded area shows the range of the STEM measured side widths of the thickest side and the corresponding range of calculated energies.

\section{Table 2. Radial QW Geometries Used for Simulations ${ }^{a}$}

$\begin{array}{cllllll}\text { geometry } & \text { facet } 1 & \text { facet } 2 & \text { facet } 3 & \text { facet } 4 & \text { facet } 5 & \text { facet } 6 \\ \mathbf{1} & d-0.5 x & d-x & d-0.5 x & d+0.5 x & d+x & d+0.5 x \\ 2 & d & d-0.5 x & d-x & d+0.5 x & d+x\end{array}$

${ }^{a}$ Both geometries are based on STEM analysis. Geometry 1 represents a mirror symmetric system with two QWs of different width next two the thickest one, whereas geometry 2 is an asymmetric case with two differently thick QWs next to the thickest one. Here, $d$ and $x$ are the mean value of the side width and thickness variation parameter, respectively.

In order to gain deeper understanding of the nature of the emission from the radial QW, we study theoretically the exciton energy levels and localization. We use an approach established in previous works for polygonal quantum rings and prismatic shells ${ }^{37,38,40,41}$ but restrict ourselves to the crosssectional plane at a given point along the $\mathrm{NW}$ axis. In particular, we calculate the single-particle states with a discretization method based on a polar grid, which is further adopted to the hexagonal geometries obtained from the crosssectional STEM images of the NWs [cf. Figure 1 and Table 1]. Next, we use these states to construct the electron-hole Hamiltonian with the Coulomb interaction included.

We start with an ideal NW geometry with a perfectly symmetric hexagonal cross section of the radial QW. We set the mean QW thickness $(d)$ and the external radius $\left(R_{\text {ext }}\right)$ equal to 5.5 and $80 \mathrm{~nm}$, respectively. Next, we vary the thickness of the radial QW on different facets in two ways as depicted in Figure 4a and shown in Table 2. Instead of accounting for the complex combination of thickness fluctuations and deformation of the perfect hexagonal shape observed by STEM, we restrict ourselves to the two cases of minimal deformation and a linearly graded thickness variation. In the case of geometry 1 , the thickest facet $\left(d_{5}\right)$ is situated next to two equally narrow, yet still thick facets and opposite to the thinnest one, thus preserving a mirror symmetry. For geometry 2, the mirror symmetry is also lifted, and the thickest facet is only neighbored by two facets with QWs of different width. In addition, the thickest and thinnest facets are separated by at least one facet on each side, i.e., the side thickness changes monotonically around the circumference as observed by STEM. To quantify these deviations, we introduce a thickness variation parameter $x$ defined as $x=d_{\max }-d$. The
QW thicknesses for both geometries are summarized in Table 2 . An increase of $x$ results, on the one hand, in an increase of the thickness of QWs on one side of the hexagon and, on the other hand, in a reduction of the QW thickness for the other side of the hexagon.

The probability distributions of electrons are shown in Figure $4 \mathrm{~b}$ for both geometries for different thicknesses of the widest facet, i.e., for $d_{5}=d+x$. The corresponding probability distribution of holes are qualitatively similar. As already predicted in previous theoretical works ${ }^{10,39,65}$ for the undeformed, i.e., symmetric, QW ring structure, the probability distribution corresponding to the ground state is equally distributed between all six corners of the hexagonal cross section. Introducing a finite asymmetry results in a strong impact on the probability distribution in the QW: when increasing $d_{5}$ by only $x=0.1 \mathrm{~nm}$ corresponding to 0.5 monolayers (MLs), our calculations predict a lifting of the 6fold symmetry. Remarkably, this minute difference in thickness of about one percent between facet 5 and its thickest neighboring facet creates a 2 -fold symmetric corner state at the ends of the widest QW formed on facet 5 and a single corner state at the corner between facets 5 and 6 for geometries 1 and 2, respectively. Thus, our calculations nicely confirm that the formation of corner states with ideal 6-fold symmetry requires near-perfect 6-fold structural symmetry. Even small variations lead to complete wave function localization at the corners connecting the two widest QWs. As $x$ increases, the sharp localization peaks of the probability distribution shift toward the center of the thickest side. This results in the delocalization of the excitons from the corners toward the center of the widest QW. For $x=1 \mathrm{~nm}$ (corresponding to a relative thickness fluctuation of approx- 
imately $5 \mathrm{MLs}$ ), the exciton probability distribution is localized in the QW side facet. Thus, for the NWs studied here, $x$ ranging from $0.9 \mathrm{~nm}$ up to $1.6 \mathrm{~nm}$ compared to the mean value of $d=5.5 \mathrm{~nm}$ for a perfect hexagon, excitons do not distribute over all six corners but can be readily considered to be fully localized on the widest facet of the radial QW. Our STEM analysis shows that in realistic systems more pronounced deviations of the perfect hexagonal symmetry, e.g., deformation of the perfect hexagonal shape, occur. These are expected to enhance localization of the excitons on the widest facets. In this sense, the two geometries chosen can be considered as representative examples. Furthermore, we calculated the exciton energy as a function of $x$ for both geometries. The results are plotted in Figure 4c. As expected, the ground state exciton energies exhibit a clear red shift with increasing $x$, as for regular, planar QW structures. ${ }^{66}$ Moreover, the nearly perfect overlap of the energies obtained for the two geometries (Figure 4c) confirms that the ground state energy does not depend on the shape of the cross section, i.e., if the widest side is sufficiently thicker than the second widest side, then the ground state excitonic energy is defined solely by the width of the thickest side. We compare the results of these calculations to our experimental data. The blue shaded area in Figure $4 \mathrm{c}$ indicates the energy region of the QW emissions of the NW 4, 5 , and 6 and the corresponding increase of QW thickness compared to the symmetric case. From this comparison, we may conclude that the thickness deviations of both QW segments are approximately 1.4 and $3.8 \mathrm{~nm}$, respectively. These values exceed those obtained from STEM analysis ranging from 0.9 to $1.6 \mathrm{~nm}$. For comparison, the red colored area in Figure $4 c$ indicates the exciton energy levels expected from the QW thicknesses obtained from our STEM analysis. Considering the approximations applied in the model, in particular the infinite energy barriers, the overestimation of the exciton ground state with respect to the well thickness is expected. Therefore, the results of our calculations can be considered in good agreement with our experimental findings.

In conclusion, the results presented here have multiple important implications for the realization of functional coreshell NW heterostructures with embedded quantum nanostructures. We investigated the structural, optical, and electronic properties of a hexagonal QW system embedded in commonly grown $\mathrm{GaAs} / \mathrm{Al}_{0.3} \mathrm{Ga}_{0.7} \mathrm{As}$ core/shell $\mathrm{NW}$ heterostructure. Our results show that a small distortion of the perfect hexagonal shape of the radial QW leads to a significant change of the optical and electronic characteristics. Moreover, small variations of the QW thickness on the sidewall facets strongly affect the localization of the charge carrier distribution. In particular, the 6-fold corner states-like symmetry of a perfect hexagonal shape is rapidly lifted and a localization fully at the side facets takes place, which in turn results in a change of the optical and electrical properties. These moderate deviations from the ideal symmetric hexagonal geometry result in clear spectral shifts and multipeak structure of the PL spectrum of the radial QW. Furthermore, the well width fluctuations lead to formation of spatially separated but interconnected QW systems. Our acousto-optoelectric experiments also directly prove mutual coupling and reversible carrier exchange between the two neighboring QW systems on subnanosecond time scales.

Thus, the perfection down to the monolayer accuracy required to create the often predicted highly symmetric quantum states defines one great challenge for future experiments. Alternative routes may be derived from tailoring composition-driven faceting ${ }^{28}$ with elegant growth modulation techniques developed for vicinal wafer-scale substrates, for instance, the serpentine superlattice, ${ }^{67,68}$ and pave the way toward one-dimensional quantum confined systems on the one-dimensional NW geometry.

\section{AUTHOR INFORMATION}

\section{Corresponding Authors}

*E-mail: hubert.krenner@physik.uni-augsburg.de.

*E-mail: anna.sitek@pwr.edu.pl.

ORCID $\odot$

Anna Sitek: 0000-0002-0602-1959

Gregor Koblmüller: 0000-0002-7228-0158

Hubert J. Krenner: 0000-0002-0696-456X

Author Contributions

${ }^{\nabla}$ M.M.S. and A.S. contributed equally.

Notes

The authors declare no competing financial interest.

\section{ACKNOWLEDGMENTS}

This work was supported by the Deutsche Forschungsgemeinschaft (DFG) via Research grants KR3790/6-1 and KO4005/ 6-1, the Excellence Initiative of the German Federal Government via the Cluster of Excellence Nanosystems Initiative Munich (NIM), and the Icelandic Research Fund within project No. 163438-051.

\section{REFERENCES}

(1) Capasso, F. Band-Gap Engineering: From Physics and Materials to New Semiconductor Devices. Science 1987, 235 (4785), 172-176.

(2) Gudiksen, M. S.; Lauhon, L. J.; Wang, J.; Smith, D. C.; Lieber, C. M. Growth of Nanowire Superlattice Structures for Nanoscale Photonics and Electronics. Nature 2002, 415 (6872), 617-620.

(3) Lauhon, L. J.; Gudiksen, M. S.; Wang, D.; Lieber, C. M. Epitaxial Core-shell and Core-multishell Nanowire Heterostructures. Nature 2002, 420 (6911), 57-61.

(4) Tomioka, K.; Motohisa, J.; Hara, S.; Hiruma, K.; Fukui, T. GaAs/AlGaAs Core Multishell Nanowire-Based Light-Emitting Diodes on Si. Nano Lett. 2010, 10 (5), 1639-1644.

(5) Tomioka, K.; Yoshimura, M.; Fukui, T. A III-V Nanowire Channel on Silicon for High-Performance Vertical Transistors. Nature 2012, 488 (7410), 189-192.

(6) Krogstrup, P.; Jørgensen, H. I.; Heiss, M.; Demichel, O.; Holm, J. V.; Aagesen, M.; Nygard, J.; Fontcuberta i Morral, A. SingleNanowire Solar Cells beyond the Shockley-Queisser Limit. Nat. Photonics 2013, 7 (4), 306-310.

(7) Mayer, B.; Rudolph, D.; Schnell, J.; Morkötter, S.; Winnerl, J.; Treu, J.; Müller, K.; Bracher, G.; Abstreiter, G.; Koblmüller, G.; et al. Lasing from Individual GaAs-AlGaAs Core-Shell Nanowires up to Room Temperature. Nat. Commun. 2013, 4, 2931.

(8) Saxena, D.; Mokkapati, S.; Parkinson, P.; Jiang, N.; Gao, Q.; Tan, H. H.; Jagadish, C. Optically Pumped Room-Temperature GaAs Nanowire Lasers. Nat. Photonics 2013, 7 (12), 963-968.

(9) Qian, F.; Li, Y.; Gradečak, S.; Park, H. G.; Dong, Y.; Ding, Y.; Wang, Z. L.; Lieber, C. M. Multi-Quantum-Well Nanowire Heterostructures for Wavelength-Controlled Lasers. Nat. Mater. 2008, 7 (9), 701-706.

(10) Fickenscher, M.; Shi, T.; Jackson, H. E.; Smith, L. M.; YarrisonRice, J. M.; Zheng, C.; Miller, P.; Etheridge, J.; Wong, B. M.; Gao, Q.; et al. Optical, Structural, and Numerical Investigations of GaAs/ AlGaAs Core-Multishell Nanowire Quantum Well Tubes. Nano Lett. 2013, 13 (3), 1016-1022.

(11) Dimakis, E.; Jahn, U.; Ramsteiner, M.; Tahraoui, A.; Grandal, J.; Kong, X.; Marquardt, O.; Trampert, A.; Riechert, H.; Geelhaar, L. 
Coaxial Multishell (In,Ga)As/GaAs Nanowires for near-Infrared Emission on Si Substrates. Nano Lett. 2014, 14 (5), 2604-2609.

(12) Stettner, T.; Zimmermann, P.; Loitsch, B.; Döblinger, M.; Regler, A.; Mayer, B.; Winnerl, J.; Matich, S.; Riedl, H.; Kaniber, M.; et al. Coaxial GaAs-AlGaAs Core-Multishell Nanowire Lasers with Epitaxial Gain Control. Appl. Phys. Lett. 2016, 108 (1), 011108.

(13) Irber, D. M.; Seidl, J.; Carrad, D. J.; Becker, J.; Jeon, N.; Loitsch, B.; Winnerl, J.; Matich, S.; Döblinger, M.; Tang, Y.; et al. Quantum Transport and Sub-Band Structure of Modulation-Doped GaAs/AlAs Core-Superlattice Nanowires. Nano Lett. 2017, 17 (8), 4886-4893.

(14) Stettner, T.; Thurn, A.; Döblinger, M.; Hill, M. O.; Bissinger, J.; Schmiedeke, P.; Matich, S.; Kostenbader, T.; Ruhstorfer, D.; Riedl, $\mathrm{H}$.; et al. Tuning Lasing Emission toward Long Wavelengths in GaAs(In,Al)GaAs Core-Multishell Nanowires. Nano Lett. 2018, 18 (10), 6292-6300.

(15) Saxena, D.; Jiang, N.; Yuan, X.; Mokkapati, S.; Guo, Y.; Tan, H. H.; Jagadish, C. Design and Room-Temperature Operation of GaAs/ AlGaAs Multiple Quantum Well Nanowire Lasers. Nano Lett. 2016, 16 (8), 5080-5086.

(16) Erhard, N.; Zenger, S.; Morkötter, S.; Rudolph, D.; Weiss, M.; Krenner, H. J.; Karl, H.; Abstreiter, G.; Finley, J. J.; Koblmüller, G.; et al. Ultrafast Photodetection in the Quantum Wells of Single AlGaAs/GaAs-Based Nanowires. Nano Lett. 2015, 15 (10), 68696874.

(17) Morkötter, S.; Jeon, N.; Rudolph, D.; Loitsch, B.; Spirkoska, D.; Hoffmann, E.; Döblinger, M.; Matich, S.; Finley, J. J.; Lauhon, L. J.; et al. Demonstration of Confined Electron Gas and Steep-Slope Behavior in Delta-Doped GaAs-AlGaAs Core-Shell Nanowire Transistors. Nano Lett. 2015, 15 (5), 3295-3302.

(18) Sakaki, H.; Noda, T.; Hirakawa, K.; Tanaka, M.; Matsusue, T. Interface Roughness Scattering in GaAs/AlAs Quantum Wells. Appl. Phys. Lett. 1987, 51 (23), 1934-1936.

(19) Herman, M. A.; Bimberg, D.; Christen, J. Heterointerfaces in Quantum Wells and Epitaxial Growth Processes: Evaluation by Luminescence Techniques. J. Appl. Phys. 1991, 70 (2), R1.

(20) Campman, K. L.; Schmidt, H.; Imamoglu, A.; Gossard, A. C. Interface Roughness and Alloy-Disorder Scattering Contributions to Intersubband Transition Linewidths. Appl. Phys. Lett. 1996, 69 (17), 2554-2556.

(21) Ruf, T.; Spitzer, J.; Sapega, V. F.; Belitsky, V. I.; Cardona, M.; Ploog, K. Interface Roughness and Homogeneous Linewidths in Quantum Wells and Superlattices Studied by Resonant AcousticPhonon Raman Scattering. Phys. Rev. B: Condens. Matter Mater. Phys. 1994, 50 (3), 1792-1806.

(22) Rudolph, D.; Funk, S.; Döblinger, M.; Morkötter, S.; Hertenberger, S.; Schweickert, L.; Becker, J.; Matich, S.; Bichler, M.; Spirkoska, D.; et al. Spontaneous Alloy Composition Ordering in GaAs-AlGaAs Core-shell Nanowires. Nano Lett. 2013, 13 (4), 15221527.

(23) Heiss, M.; Fontana, Y.; Gustafsson, A.; Wüst, G.; Magen, C.; O’Regan, D. D.; Luo, J. W.; Ketterer, B.; Conesa-Boj, S.; Kuhlmann, A. V.; et al. Self-Assembled Quantum Dots in a Nanowire System for Quantum Photonics. Nat. Mater. 2013, 12 (5), 439-444.

(24) Weiß, M.; Kinzel, J. B.; Schülein, F. J. R.; Heigl, M.; Rudolph, D.; Morkötter, S.; Döblinger, M.; Bichler, M.; Abstreiter, G.; Finley, J. J.; et al. Dynamic Acoustic Control of Individual Optically Active Quantum Dot-like Emission Centers in Heterostructure Nanowires. Nano Lett. 2014, 14 (5), 2256-2264.

(25) Mancini, L.; Fontana, Y.; Conesa-Boj, S.; Blum, I.; Vurpillot, F.; Francaviglia, L.; Russo-Averchi, E.; Heiss, M.; Arbiol, J.; Fontcuberta I Morral, A.; et al. Three-Dimensional Nanoscale Study of $\mathrm{Al}$ Segregation and Quantum Dot Formation in GaAs/AlGaAs CoreShell Nanowires. Appl. Phys. Lett. 2014, 105 (24), 243106.

(26) Jeon, N.; Loitsch, B.; Morkoetter, S.; Abstreiter, G.; Finley, J.; Krenner, H. J.; Koblmueller, G.; Lauhon, L. J. Alloy Fluctuations Act as Quantum Dot-like Emitters in GaAs-AlGaAs Core-Shell Nanowires. ACS Nano 2015, 9 (8), 8335-8343.
(27) Pöpsel, C.; Becker, J.; Jeon, N.; Döblinger, M.; Stettner, T.; Gottschalk, Y. T.; Loitsch, B.; Matich, S.; Altzschner, M.; Holleitner, A. W.; et al. He-Ion Microscopy as a High-Resolution Probe for Complex Quantum Heterostructures in Core-Shell Nanowires. Nano Lett. 2018, 18 (6), 3911-3919.

(28) Jeon, N.; Ruhstorfer, D.; Döblinger, M.; Matich, S.; Loitsch, B.; Koblmüller, G.; Lauhon, L. Connecting Composition-Driven Faceting with Facet-Driven Composition Modulation in GaAs-AlGaAs CoreShell Nanowires. Nano Lett. 2018, 18 (8), 5179-5185.

(29) Weiß, M.; Schülein, F. J. R.; Kinzel, J. B.; Heigl, M.; Rudolph, D.; Bichler, M.; Abstreiter, G.; Finley, J. J.; Wixforth, A.; Koblmüller, G.; et al. Radio Frequency Occupancy State Control of a Single Nanowire Quantum Dot. J. Phys. D: Appl. Phys. 2014, 47 (39), 394011.

(30) Shi, T.; Jackson, H. E.; Smith, L. M.; Jiang, N.; Gao, Q.; Tan, H. H.; Jagadish, C.; Zheng, C.; Etheridge, J. Emergence of Localized States in Narrow GaAs/AlGaAs Nanowire Quantum Well Tubes. Nano Lett. 2015, 15 (3), 1876-1882.

(31) Shi, T.; Jackson, H. E.; Smith, L. M.; Jiang, N.; Tan, H. H.; Jagadish, C. Thermal Delocalization of Excitons in GaAs/AlGaAs Quantum Well Tube Nanowires. Nano Lett. 2016, 16 (2), 13921397.

(32) Zheng, C.; Wong-Leung, J.; Gao, Q.; Tan, H. H.; Jagadish, C.; Etheridge, J. Polarity-Driven 3-Fold Symmetry of GaAs/AlGaAs Core Multishell Nanowires. Nano Lett. 2013, 13 (8), 3742-3748.

(33) Funk, S.; Royo, M.; Zardo, I.; Rudolph, D.; Morkötter, S.; Mayer, B.; Becker, J.; Bechtold, A.; Matich, S.; Döblinger, M.; et al. High Mobility One- and Two- Dimensional Electron Systems in Nanowire-Based Quantum Heterostructures. Nano Lett. 2013, 13 (4), 6189-6196.

(34) Davies, C. L.; Parkinson, P.; Jiang, N.; Boland, J. L.; ConesaBoj, S.; Hoe Tan, H.; Jagadish, C.; Herz, L. M.; Johnston, M. B. Low Ensemble Disorder in Quantum Well Tube Nanowires. Nanoscale 2015, 7 (48), 20531-20538.

(35) Uccelli, E.; Arbiol, J.; Morante, J. R.; Fontcuberta I Morral, A. InAs Quantum Dot Arrays Decorating the Facets of GaAs Nanowires. ACS Nano 2010, 4 (10), 5985-5993.

(36) Jadczak, J.; Plochocka, P.; Mitioglu, A.; Breslavetz, I.; Royo, M.; Bertoni, A.; Goldoni, G.; Smolenski, T.; Kossacki, P.; Kretinin, A.; et al. Unintentional High-Density p-Type Modulation Doping of a GaAs/AlAs Core-Multishell Nanowire. Nano Lett. 2014, 14 (5), 2807-2814

(37) Sitek, A.; Ţolea, M.; Niţă, M.; Serra, L.; Gudmundsson, V.; Manolescu, A. In-Gap Corner States in Core-Shell Polygonal Quantum Rings. Sci. Rep. 2017, 7, 40197.

(38) Sitek, A.; Urbaneja Torres, M.; Torfason, K.; Gudmundsson, V.; Bertoni, A.; Manolescu, A. Excitons in Core-shell Nanowires with Polygonal Cross Sections. Nano Lett. 2018, 18 (4), 2581-2589.

(39) Ballester, A.; Planelles, J.; Bertoni, A. Multi-Particle States of Semiconductor Hexagonal Rings: Artificial Benzene. J. Appl. Phys. 2012, 112 (10), 104317.

(40) Sitek, A.; Serra, L.; Gudmundsson, V.; Manolescu, A. Electron Localization and Optical Absorption of Polygonal Quantum Rings. Phys. Rev. B: Condens. Matter Mater. Phys. 2015, 91 (23), 235429.

(41) Sitek, A.; Thorgilsson, G.; Gudmundsson, V.; Manolescu, A. Multi-Domain Electromagnetic Absorption of Triangular Quantum Rings. Nanotechnology 2016, 27 (22), 225202.

(42) Fontcuberta I Morral, A.; Colombo, C.; Abstreiter, G.; Arbiol, J.; Morante, J. R. Nucleation Mechanism of Gallium-Assisted Molecular Beam Epitaxy Growth of Gallium Arsenide Nanowires. Appl. Phys. Lett. 2008, 92 (6), 063112.

(43) Colombo, C.; Spirkoska, D.; Frimmer, M.; Abstreiter, G.; Fontcuberta i Morral, A. Ga-Assisted Catalyst-Free Growth Mechanism of GaAs Nanowires by Molecular Beam Epitaxy. Phys. Rev. B: Condens. Matter Mater. Phys. 2008, 77 (15), 155326.

(44) Hertenberger, S.; Rudolph, D.; Bichler, M.; Finley, J. J.; Abstreiter, G.; Koblmüller, G. Growth Kinetics in Position-Controlled and Catalyst-Free InAs Nanowire Arrays on $\mathrm{Si}(111)$ Grown by 
Selective Area Molecular Beam Epitaxy. J. Appl. Phys. 2010, 108 (11), 114316.

(45) Mårtensson, T.; Svensson, C. P. T.; Wacaser, B. A.; Larsson, M. W.; Seifert, W.; Deppert, K.; Gustafsson, A.; Wallenberg, L. R.; Samuelson, L. Epitaxial III-V Nanowires on Silicon. Nano Lett. 2004, 4 (10), 1987-1990.

(46) Joyce, H. J.; Gao, Q.; Tan, H. H.; Jagadish, C.; Kim, Y. TwinFree Uniform Epitaxial GaAs Nanowires Grown by a TwoTemperature Process. Nano Lett. 2007, 7 (4), 921-926.

(47) Russo-Averchi, E.; Heiss, M.; Michelet, L.; Krogstrup, P.; Nygard, J.; Magen, C.; Morante, J. R.; Uccelli, E.; Arbiol, J.; Fontcuberta i Morral, A. Suppression of Three Dimensional Twinning for a $100 \%$ Yield of Vertical GaAs Nanowires on Silicon. Nanoscale 2012, 4 (5), 1486.

(48) Rudolph, D.; Hertenberger, S.; Bolte, S.; Paosangthong, W.; Spirkoska, D.; Döblinger, M.; Bichler, M.; Finley, J. J.; Abstreiter, G.; Koblmüller, G. Direct Observation of a Noncatalytic Growth Regime for GaAs Nanowires. Nano Lett. 2011, 11 (9), 3848-3854.

(49) Sibirev, N. V.; Tchernycheva, M.; Timofeeva, M. A.; Harmand, J. C.; Cirlin, G. E.; Dubrovskii, V. G. Influence of Shadow Effect on the Growth and Shape of InAs Nanowires. J. Appl. Phys. 2012, 111, 104317.

(50) Rudolph, D.; Schweickert, L.; Morkötter, S.; Loitsch, B.; Hertenberger, S.; Becker, J.; Bichler, M.; Abstreiter, G.; Finley, J. J.; Koblmüller, G. Effect of Interwire Separation on Growth Kinetics and Properties of Site-Selective GaAs Nanowires. Appl. Phys. Lett. 2014, 105 (3), 033111.

(51) Dubrovskii, V. G.; Xu, T.; Díaz Álvarez, A.; Plissard, S. R.; Caroff, P.; Glas, F.; Grandidier, B. Self-Equilibration of the Diameter of Ga-Catalyzed GaAs Nanowires. Nano Lett. 2015, 15 (8), 55805584.

(52) Rudolph, D.; Schweickert, L.; Morkötter, S.; Hanschke, L.; Hertenberger, S.; Bichler, M.; Koblmüller, G.; Abstreiter, G.; Finley, J. J. Probing the Trapping and Thermal Activation Dynamics of Excitons at Single Twin Defects in GaAs-AlGaAs Core-Shell Nanowires. New J. Phys. 2013, 15, 113032.

(53) Wang, W. I. Instabilities of (110) III-V Compounds Grown by Molecular Beam Epitaxy. J. Vac. Sci. Technol., B: Microelectron. Process. Phenom. 1983, 1 (3), 630.

(54) Brunner, K.; Abstreiter, G.; Böhm, G.; Tränkle, G.; Weimann, G. Sharp-Line Photoluminescence of Excitons Localized at GaAs/ AlGaAs Quantum Well Inhomogeneities. Appl. Phys. Lett. 1994, 64 (24), 3320-3322.

(55) Leosson, K.; Jensen, J. R.; Langbein, W.; Hvam, J. M. Exciton Localization and Interface Roughness in Growth-Interrupted GaAs/ AlAs Quantum Wells. Phys. Rev. B: Condens. Matter Mater. Phys. 2000, 61 (15), 10322-10329.

(56) Yoshita, M.; Kondo, N.; Sakaki, H.; Baba, M.; Akiyama, H. Large Terrace Formation and Modulated Electronic States in (110) GaAs Quantum Wells. Phys. Rev. B: Condens. Matter Mater. Phys. 2001, 63 (7), 075305.

(57) Perera, S.; Fickenscher, M. A.; Jackson, H. E.; Smith, L. M.; Yarrison-Rice, J. M.; Joyce, H. J.; Gao, Q.; Tan, H. H.; Jagadish, C.; Zhang, X.; et al. Nearly Intrinsic Exciton Lifetimes in Single TwinFree GaAs/AlGaAs Core-Shell Nanowire Heterostructures. Appl. Phys. Lett. 2008, 93 (5), 053110.

(58) Demichel, O.; Heiss, M.; Bleuse, J.; Mariette, H.; Fontcuberta i Morral, A. Impact of Surfaces on the Optical Properties of GaAs Nanowires. Appl. Phys. Lett. 2010, 97 (20), 201907.

(59) Kinzel, J. B.; Rudolph, D.; Bichler, M.; Abstreiter, G.; Finley, J. J.; Koblmüller, G.; Wixforth, A.; Krenner, H. J. Directional and Dynamic Modulation of the Optical Emission of an Individual GaAs Nanowire Using Surface Acoustic Waves. Nano Lett. 2011, 11 (4), $1512-1517$.

(60) Kinzel, J. B.; Schülein, F. J. R.; Weiß, M.; Janker, L.; Bühler, D. D.; Heigl, M.; Rudolph, D.; Morkötter, S.; Döblinger, M.; Bichler, M.; et al. The Native Material Limit of Electron and Hole Mobilities in Semiconductor Nanowires. ACS Nano 2016, 10 (5), 4942-4953.
(61) Alsina, F.; Santos, P. V.; Hey, R.; García-Cristóbal, A.; Cantarero, A. Dynamic Carrier Distribution in Quantum Wells Modulated by Surface Acoustic Waves. Phys. Rev. B: Condens. Matter Mater. Phys. 2001, 64 (4), 041304.

(62) Schülein, F. J. R.; Pustiowski, J.; Müller, K.; Bichler, M.; Koblmüller, G.; Finley, J. J.; Wixforth, A.; Krenner, H. J. Surface Acoustic Wave Controlled Charge Dynamics in a Thin InGaAs Quantum Well. JETP Lett. 2012, 95 (11), 575-580.

(63) Büyükköse, S.; Hernández-Mínguez, A.; Vratzov, B.; Somaschini, C.; Geelhaar, L.; Riechert, H.; Van Der Wiel, W. G.; Santos, P. V. High-Frequency Acoustic Charge Transport in GaAs Nanowires. Nanotechnology 2014, 25 (13), 135204.

(64) García-Cristóbal, A.; Cantarero, A.; Alsina, F.; Santos, P. V. Spatiotemporal Carrier Dynamics in Quantum Wells under Surface Acoustic Waves. Phys. Rev. B: Condens. Matter Mater. Phys. 2004, 69 (20), 205301.

(65) Ferrari, G.; Goldoni, G.; Bertoni, A.; Cuoghi, G.; Molinari, E. Magnetic States in Prismatic Core Multishell Nanowires. Nano Lett. 2009, 9 (4), 1631-1635.

(66) Miller, R. C.; Kleinman, D. A.; Gossard, A. C. Energy-Gap Discontinuities and Effective Masses for GaAs- $\mathrm{Al}_{x} \mathrm{Ga}_{1-\mathrm{x}} \mathrm{As}$ Quantum Wells. Phys. Rev. B: Condens. Matter Mater. Phys. 1984, 29 (12), 7085-7087.

(67) Miller, M. S.; Weman, H.; Pryor, C. E.; Krishnamurthy, M.; Petroff, P. M.; Kroemer, H.; Merz, J. L. Serpentine Superlattice Quantum-Wire Arrays of $(\mathrm{Al}, \mathrm{Ga})$ As Grown on Vicinal GaAs Substrates. Phys. Rev. Lett. 1992, 68 (23), 3464-3467.

(68) Hu, S.-Y.; Yi, J. C.; Miller, M. S.; Leonard, D.; Young, D. B.; Gossard, A. C.; Dagli, N.; Petroff, P. M.; Coldren, L. A. Serpentine Superlattice Nanowire-Array Lasers. IEEE J. Quantum Electron. 1995, 31 (8), 1380-1388. 